\title{
Small Blob Detection and Classification in 3D MRI Human Kidney Images Using IMBKM and EDCNN Classifier
}

\author{
Sitanaboina S L Parvathi , Dr. Harikiran Jonnadula \\ 1 esearch Scholar, School of Computer Science and Engineering, Vellore Institute of Technology, VIT-AP \\ University, Vijayawada, \\ 2Assistant Professor, School of Computer Science and Engineering, Vellore Institute of Technology, VIT-AP. \\ s.srilakshmiparvathi@gmail.com
}

Article History: Received: 11 January 2021; Accepted: 27 February 2021; Published online: 5 April 2021

\begin{abstract}
The spatial and temporal resolution is dramatically increased due to the quick development of medical imaging technology, which in turn increases the size of clinical imaging data. Typically, it is very challenging to do small blob segmentation as of Medical Images (MI) but it encompasses so many vital applications. Some examples are labelling cell, lesion, along with glomeruli aimed at disease diagnosis. Though various detectors were suggested by the prevailing method for this type of issue, they mostly used 2D detectors, which may render less detection accuracy. To trounce this, the system has developed an efficient small Blob Detection (BD)as well as classification in 3D Magnetics Resonance Imaging (MRI) human kidney images utilizingImproved Mini Batch K-Means (IMBKM)and Enhanced Deep Convolutionals Neural Network (EDCNN) classifier. To segment the blob portions, the image is first ameliorated via Enhanced Contrast Limited Adaptive Histogram Equalization (ECLAHE) followed by the IMBKM algorithm. After that, to determine the segmentation performance, the pixels' percentage in the detected blob portion is gauged. In addition, statistical, GLCM, together with shape features are extracted as of the segmented blob potions. Lastly, the EDCNN takes care of the classification, which classifies '4' classes, say, Normal, Glomerulonephritis, Stone, and Pyelonephritis. The experimental outcomes exhibit that IMBKM and EDCNN have the potential to automatically detect blobs and classify the blobs efficiently than the top-notch methods.

Keywords: Magnetic Resonance Imaging (MRI), Enhanced Contrast Limited Adaptive Histogram Equalization (ECLAHE), Improved Mini Batch K-Means (IMBKM), Gray-Level Co-occurrence Matrix (GLCM), and Enhanced Deep Convolutional Neural Network (EDCNN).
\end{abstract}

\section{INTRODUCTION}

Individualized precision medicine has appeared as a novel paradigm for the diagnosis along with treatment in healthcare in the precedent decennium. Medical imaging is a cornerstone for precision medicine [1, 2]. A significant tool for investigating kidney microstructure is MRI [3, 4]. Molecular MRI is a new field that develops tools for localizing blobs in kidney MRI images. Multiple objects in images display a blob-like semblance, and therefore, BD has a broad range of applications, namely cell counting, bubble extraction, plane detection, quantum dot recognition [5], etc. In a heterogeneous image, high-resolution object detection is vital for acquiring meaningful information. Blobs (also called particles [6] or dots) can be specified as small structures whose visual properties, namely brightness or color, are different from those in their neighboring area. Lower image resolution along with high image noise is the major challenges for detecting blobs. Multiple small blobs can overlap among themselves.

The pipeline of $\mathrm{BD}$ was split into ' 3 ' steps: (i) image smoothing (ii) feature vector extraction; and (iii) blob identification in the beginning, before the deep learning era [7]. Although high-quality quality images are generated by the systems during image smoothing after a while, it is tough to predict an accurate time and an appropriate number of images for attaining higher-quality MRI images. Therefore, finding quality images is tedious along with labor-intensive [8]. Several researchers proposed many Image Enhancement (IE)techniques [9], namely, the Contrasts Limited Adaptive Histograms Equalization (CLAHE), Adjust Intensity Values (AIV), the Riesz fractional [10], the Histogram Equalizations along with Tsallis entropy technique [11]. The AIV, CLAHE Riesz fractional, and Tsallis entropy failed to enhance the edge details of the kidney contrasted to the input image. So, an effective IE technique is employed by the proposed technique. Next, only a few features are extracted from the enhanced images to detect accurately the results, however, an accurate result was not given by taking a few features. BD is a vital step in which the functions are explained in the above-stated statements. The most frequent method proposed by researchers among various approaches for BD is thresholding [12]. Thresholding is centered on the supposition that the blob intensity has varied significantly when contrasted to the background intensity. But, this supposition might not be right and can bring about the wrong detection of objects [13]. Recently, many techniques, like Laplacians of Gaussian (LoG) [14], Difference-of-Gaussians (DoG), Determinant of Hessian (DoH) [15], generalized-gLoG (gLoG) [16], and Hessian-centeredDoG (HDoG) have been suggested in the last decades [17], which precisely detected and delineated blob shapes. However, the applications of these transformations are limited by the computational encumber in 3D BD. Thus, an effective IE 
method and deep convolutionals neural network (DCNN) algorithm for efficiently detecting the blobs in 3D MRI human kidney images is proposed by this paper.

This paper is categorized as: Section 2 offers the total review of the proposed BD in 3D MRI human kidney images. Section 3 elaborates the details of the proposed BD in 3D MRI human kidney images. Section 4 considers and elucidates the experimental outcomes. Section 5 explains the conclusions as well as future possibilities of the proposed work.

\section{RELATED WORK}

Min Zhang et al. [18] offered a computationally effective algorithm, named the HDoG, for segmenting small blobs (for instance, kidney's glomeruli) as of 3D MI centered upon local convexity, intensity, along with shape information. The image was initially flattened together with pre-segmented into smaller blob candidate areas centered upon local convexity. The ' 2 ' $3 \mathrm{D}$ regional features were then removed as of the candidate areas. The ' 3 ' features were utilized on an un-supervised learning algorithm aimed at auto post-pruning in tandem with regional intensity. The HDoG's performance was satisfactory in the detection of vast quantities of small blobs as showed by the results. Missed detection along with false-positive detections problem was some problems possessed. For example, discontinuity was shown by some glomeruli in the images because of the imaging acquisition artifact.

Yanzhe Xu et al. [19] presented improved small BD in 3D images centered on a jointly constrained deep learning along with hessian analysis. The system was composed of ' 3 ' steps. The DoG was initially utilized by the system for smoothing the images, followed by a Hessian analysis that was used for identifying the potential blob candidates centered on local convexity. Next, a trained U-Net was utilized for generating a Probability Map (PM) with which the probable blob locations were captured. Then, the PM was combined with the blob candidates for identifying the true blobs. The system had rendered better outcomes than the existent methodologies as revealed by the experimental outcomes. But, the supposition that the blobs were convex and same in size might not be strong for non-convex objects of different sizes.

Min Zhang et al. [20] recommended a detector named Hessian-LoG (HLoG) centered on the scale-spaces theory as a basis. An image was first flattened through the LoG like most imaging detectors. The hessian analysis was then launched for identifying the single optimal scales centered upon which a pre-segmentation was performed. So, the regional features could be recovered. The unsupervised clustering algorithm enabled by these features for post-pruning should be stronger and also responsive than the conventional threshold-centered post-pruning usually utilized in many imaging detectors. The HLoG had statistically outperformed the contrasted detectors as shown by the results. But, it was receptive to the background noises that could result in false indications.

Min Zhang et al. [21] presented a hessian-centered algorithm for kidney glomerulus detections on 3D MRI. The image was first flattened through DoG followed by the Hessian process to pre-segment and the boundary of the glomerulus candidates was outlined. A basis was provided for extracting the regional features utilized on an unsupervised clustering algorithm, the false identifications that occur in pre-segmentation are removed for completing the segmentation. The Hessian-centeredDoG had the potential for automatically detecting the glomeruli, as of MRI on 3D, which allowed measurements of renal micro-structure along with pathology in preclinical as well as clinical environments. But, the system was difficult owing to various features.

Fei Gao et al. [22] suggested a 3D small structure (also called blob) detection in the MI centered on texture analysis. For small structures segmentation, '5' textual features in 3D were extracted. Comprehensive evaluations for every feature combination on both synthetic as well as real-world 3D images were conducted by the system for testing their accuracy and applicability. ' 1 ' feature set combined texture features along with regional geometric features was selected centered on the evaluation results. This feature set contained average intensity, blobness, intensity standard deviation, together with entropy. Thus, this feature set was vigorous to noises and was capable of precisely identifying the small blobs from 3D images. But the system was not appropriate for other real datasets, like mouse or human.

Hui Kong et al. [23] introduced a gLoG filter for BD. The general 3-D LoG scales-spaces blob detector was generalized to a 5-D gLoG scales-space one in which the ' 5 ' parameters were images-domain coordinates ( $\mathrm{x}, \mathrm{y})$, scales $(\sigma \mathrm{x}, \sigma \mathrm{y})$, along with orientation $(\theta)$, correspondingly. A more viable solution was provided by means of locating the local maximas of an intermediary map, which was achieved by aggregating the log-scalesnormalized convolution responses of every individual gLoG filter rather than seeking the local extremas of the image's 5-D gLoG scales space for the location of blobs. The system had given better outcomes when contrasted with the first-rate methodologies as shown by the results, but the prediction accuracy was low when contrasted with deep learning techniques.

\section{SMALL BLOB DETECTION AND CLASSIFICATION IN 3D MRI HUMAN KIDNEY} IMAGES

Current progressions in the medical imaging technique have significantly improved the imaging-centred diagnosis that needs efficient and accurate computational algorithms for processing the images (for instance, 
measure the objects) aimed at the quantitative assessment. The identifying objects' challenges in the MRI kidney images, especially small objects named blobs, possess lower image resolution, overlap betwixt the blobs, and image noise. Few filtering techniques as well as machine learning methods are utilized aimed at identifying the blob in MRI images, however, it affects the internal noises; it consumes extra training time and offers low prediction accuracy. Consequently, this work proposed an effective technique aimed at identifying the blob present in the 3D MRI human kidney images. The proposed system encompasses '5' paces: IE, segmentation, identifying the area of blobs, feature extraction (FE), along with classification. Firstly, the inputted 3D MRI human kidney images are improved. Next, the regions possessing blobs have been segmented. Next, the percentage of pixels render in the blobs is computed aimed at identifying the segmentation performance. Then, as of the blob regions, few vital features like statistical, GLCM, and also Shape features are extracted that are fed into the classification stage. In the categorization phase, the kidney objects' classes are categorized utilizing the EDCNN. Figure 1 exhibits the proposed methodology's block diagram,

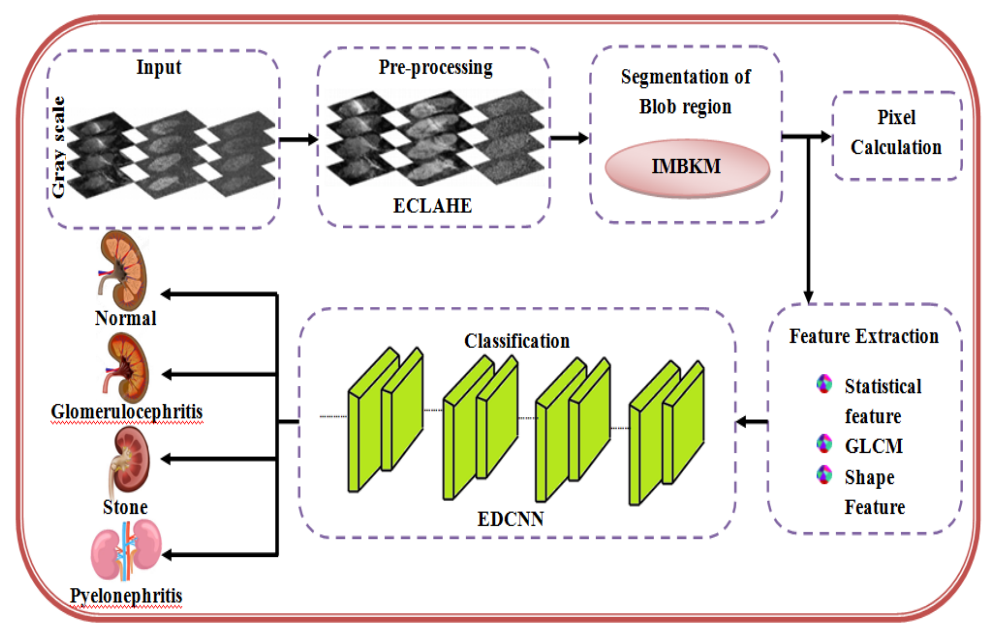

\subsection{Data Collection}

Figure 1: Architecture diagram of the system model

At first, the 3D MRI human kidney images are gathered as of the datasets that are available publically. As of the MRI sequences, the kidney MRI dataset comprises less-contrast images. It is arithmetically articulated as:

$$
\bar{A}_{k d}^{\prime}=\left(\bar{a}_{1}^{\prime}, \bar{a}_{2}^{\prime}, \bar{a}_{3}^{\prime}, \ldots \ldots \ldots \ldots \ldots . . . . . \bar{a}_{n}^{\prime}\right)
$$

Here, $\bar{A}_{k d}^{\prime}$ signifies the inputted 3D MRI human kidney dataset; $\bar{a}_{n}^{\prime}$ implies the $n$-number of images prevalent in the dataset.

\subsection{Image Enhancement using ECLAHE Algorithm}

Past the data collection as of the dataset, the IE procedure is done, which is a main step on the image processing. The 3D MRI human kidney images prevalent in the dataset have been gray-level images. It comprises just black and white pixels or gray shades. This image differs as of every other colour image since each pixel needed less information. IE increases the image's quality by incrementing the luminance difference betwixt the fore-ground and back-ground. Here, the IE operation is executed utilizing the ECLAHE algorithm. It is a histogram-centred IE technique, which restricts amplification centred on the clipping performed in the histogram aimed at restricting it till the pre-defined level. Nevertheless, it shows unnatural outcomes for images comprising large backgrounds and is not much apt aimed at the kidney images possessing very fine details. The proposed work utilizes the ECLAHE method aimed at trouncing this problem. The ECLAHE algorithm's steps are elucidated as:

Step 1: The IE technique's initial step is applying local contrast enhancement on the inputted 3D MRI human kidney images; it takes both local and global information aimed at producing the enhanced image. It is articulated as:

$$
\begin{aligned}
& \bar{D}_{f o}^{\prime \prime}=\bar{B}_{t f}^{\prime \prime} *\left(\bar{A}_{k d}^{\prime}-e_{a}\right)+e_{a} \\
& \bar{B}_{t f}^{\prime \prime}=\frac{\bar{C}_{e p}^{\prime \prime} \cdot \bar{C}_{g m}^{\prime \prime}}{\sigma}
\end{aligned}
$$

Here, $D_{f o}^{\prime \prime}$ implies the local contrast-enhanced image, $\bar{B}_{t f}^{\prime \prime}$ signifies the transformation function, $\bar{A}_{k d}^{\prime}$ implies the inputted image, $e_{a}$ symbolizes the local mean, $\bar{C}_{e p}^{\prime \prime}$ signifies the enhancement parameter, $\bar{C}_{g m}^{\prime \prime}$ implies the inputted image's global mean, $\sigma$ implies the local standard deviations. It spotlights the inputted 3D MRI human kidney images finer details. 
Step 2: Then, take the local contrast-enhanced images. As of the images, extract all the inputted values like the number of regions in a row as well as column direction, number of bins utilized in the histogram transformation function, distribution parameter type, and clip limit. The clip limit aimed at the contrast enhancement procedure is normalized as of 0 to 1 . Greater numbers obtain more contrast outcomes.

Step 3: After that, pre-process the inputs, that means the original images are split into as few regions. Here, indicate the real clip limit as of the normalized value if required, pad the image before dividing it as regions.

Step 4: Next, built gray-level mapping along with the clipped histogram. The numbers of pixels existent on the contextual region are split equally in every gray-level. Hence, the average number of pixels prevalent in the gray-level is articulated as:

$$
\bar{D}_{\text {avg }}^{\prime \prime}=\frac{E_{\left(f_{m}-F_{k}\right)}-E_{\left(f_{m}-F_{z}\right)}}{E_{G}}
$$

Here, $\bar{D}_{\text {avg }}^{\prime \prime}$ implies the average number of pixels, $E_{G}$ signifies the totalgray-level prevalent on the contextual region; $E_{\left(f_{m}-F_{k}\right)}$ symbolizes the total pixels prevalent on the contextual region's $F$ direction; $E_{\left(f_{m}-F_{z}\right)}$ implies the number of pixels existent in the contextual region's $E$ direction.

Step 5: Next, estimate the actual clip limit $\bar{G}_{a c l}^{\prime \prime}$ utilizing the eqn. below,

$$
\bar{G}_{a c l}^{\prime \prime}=E_{N C} * \bar{D}_{a v g}^{\prime \prime}
$$

Here, $E_{N C}$ signifies the number of clips.

Step 6: Interpolation permits a major enhancement in the efficiency lacking the result's quality. Centred on these procedures, the 3D MRI human kidney image's contrast is upgraded that is signified as $\bar{H}_{c e}^{\prime \prime}$.

\subsection{Segmentation of Blob Region}

Here, the blobs prevalent in the improved image have been segmented utilizing the IMBKM algorithm. In general, MBKM algorithm is the efficient algorithm aimed at segmenting little objects. Utilizing the MBKM lessens the computing time as much possible as maintaining the data accuracy. It is much simple to utilize. This algorithm's impact is a little bad analogized to the standard algorithm. Nevertheless, the MBKM executes additional clustering by carrying out in advance the related mathematical statistics on the small data batches. Consequently, the proposed system utilizes a stochastic gradient descents technique aimed at decrementing the summation of squared errors. Hence, the proposed system is termed the IMBKM algorithm, whose steps are exemplified as:

Step 1: At first, it takes $\bar{H}_{c e}^{\prime \prime}$. After that, randomly split the images into as various batches, and consider a tiny batch as whole. It is arithmetically articulated as:

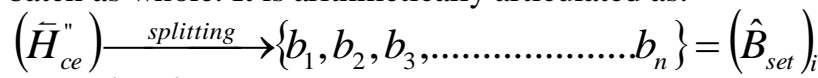

Here, $\left(\hat{B}_{\text {set }}\right)_{i}$ implies the multiple batches set, $\left\{b_{1}, b_{2}, b_{3}, \ldots \ldots \ldots \ldots \ldots \ldots . . . . . b_{n}\right\}$ signifies the multiple batches that are arbitrarily split.

Step 2: Then, the stochastic gradients descents technique is executed in various batches aimed at decrementing the computational complexity, along with the lessening of error. This is articulated as:

$$
\tilde{I}_{o p}^{\prime \prime}\left(\hat{B}_{\text {set }}\right)_{i}=\frac{1}{N} \sum_{i=1}^{n}\left(\tilde{I}_{o p}^{\prime \prime}\right)_{i}\left(\hat{B}_{\text {set }}\right)_{i}
$$

Here, $N$ signifies the multiple batches set, $\bar{I}_{o p}^{\prime \prime}\left(\hat{B}_{\text {set }}\right)$ implies the optimum values aimed at their inputted multiple batches set.

Step 3:Next, the system attains a $J_{\text {set }}$ of cluster centre $j$ for decrementing over the $\left(\hat{B}_{\text {set }}\right)_{i}$ of small-batch $j$ as signified in the eqn. (8),

$$
\operatorname{Min} \sum\left\|f\left(J_{s e t}, b_{n}\right)-b_{n}\right\|^{2}(8)
$$

Here, $f\left(J_{\text {set }}, b_{n}\right)$ symbolizes the closest cluster centre $j \in J_{\text {set }}$ to the small batch $b_{n}$.

Step 4: Centred on the equation above, iterative the cluster centre updation till the cluster centre does not vary any longer.

Step 5: Repeatedly execute the steps above till segmenting the blobs area and normal area prevalent in the contrast-enhanced images. 


\subsection{Pixel Calculation}

Past the blobs' segmentation, the proposed work identifies the detected objects' percentage aimed at identifying accurately the segmentation performance. Here, the pixel computation is carried out by taking the percentage of a number of pixels on the object detected to an image's total number of pixels. At first, it takes the inputted blob detected image (attained as of the segmentation procedure). Next, calculate the blob detected pixels' percentage as:

$$
\begin{aligned}
& \bar{L}_{T P}=\bar{M}_{A P}+\bar{M}_{N P}(9) \\
& \bar{N}_{P P}=\frac{\bar{M}_{A P}}{\overleftarrow{L}_{T P}} \times 100(10)
\end{aligned}
$$

Here, $\bar{L}_{T P}$ implies the total number of grounded pixels; $\bar{M}_{A P}$ symbolizes the blob detected regions' total pixels; $\bar{M}_{N P}$ signifies the total pixels of the normal regions; $\bar{N}_{P P}$ implies the blob detected pixels' percentage.

\subsection{Feature Extraction}

Here, a few vital features are extracted from the segmented blob portions to lessen training time. FE's key objective is to attain the major related information as of the original data and signify the information in a low dimensionality space. In this stage, as of the images (segmented), the statistical, shape features, and GLCM are extracted, which are explicated as:

\subsubsection{Statistical features}

The statistical features have been significant in the 3D MRI human kidney images. As of the images (segmented), the standard deviation, skewness, as well as kurtosis statistical features were extracted. These are elucidated as:

\section{a) Arithmetic Mean}

It is the mean of the values as of the segmented portions $o_{1}, O_{2}, O_{3}, \ldots \ldots \ldots \ldots \ldots . . ., O_{k}$ placed within a time window; it is detailed as:

$$
\mu=\frac{1}{k} \sum_{j=1}^{k} o_{j}(11)
$$

\section{b) Standard deviation}

The standard deviation $\sigma$ is computed utilizing the equation below aimed at measuring how the values $o_{1}, o_{2}, O_{3}, \ldots \ldots \ldots \ldots \ldots . . . . ., o_{k}$ are widely spread.

$\sigma=\sqrt{\frac{1}{k} \sum_{j=1}^{k}\left(o_{j}-\mu\right)^{2}}$

\section{c) Skewness}

A distribution's degree of asymmetry around its mean is characterized by means of the Skewness; it is equated as:

$$
\text { skewness }=\frac{\mu^{3}}{\sigma^{3}}(13)
$$

Here, $\mu^{3}$ signifies the $3^{\text {th }}$ moment concerning the mean; $\sigma^{3}$ implies the $3^{\text {th }}$ moment concerning the $\sigma$.

\section{d) Kurtosis}

The kurtosis also is a no dimensional quantity that measures a distribution's relative flatness or peakedness. It is equated as:

$$
\text { kurtosis }=\frac{\mu^{4}}{\sigma^{4}}(14)
$$

Here, $\mu^{4}$ and $\sigma^{4}$ signifies the $4^{\text {th }}$ moment concerning the mean and $\sigma$.

\subsubsection{Gray-Level Co-occurrence Matrix (GLCM)}

It utilizes a statistical technique aimed at evaluating the texture, pondering the pixels' spatial relationship. The GLCM functions are utilized aimed at identifying a segmented image's texture properties by computing the occurrence frequency of pixel pairs with certain values as well as in a specific spatial relationship. As of the segmented blob portions, the energy, entropy, together with homogeneity features are extracted. The features are detailed as: 


\section{a) Energy}

It is defined grounded on the block's normalized histogram.

Energy $=\frac{1}{u \times v} \sum_{a=1}^{u} \sum_{b=1}^{v}(Z(a, b))^{2}(15)$

Here, $\sum_{a=1}^{u} \sum_{b=1}^{v} Z(a, b)$ signifies the addition of every pixel values of the block and $u \times v$ signifies the frame's size

\section{b) Entropy}

A statistical measure of randomness which can be utilized for characterizing the block's texture is termed Entropy.

$$
\text { Entropy }=\frac{1}{u \times v} \sum_{a=1}^{u} \sum_{b=1}^{v}(Z(a, b)) \log _{2}(Z(a, b))
$$

\section{c) Homogeneity}

It is the closeness of the elements' distribution on the GLCM towards the GLCM diagonal.

$$
\text { Homogeneity }=\sum_{a=1}^{u} \sum_{b=1}^{v} \frac{(Z(a, b))}{1+(a-b)^{2}}(17)
$$

\subsubsection{Shape Feature (SF)}

SFs are the visual features of the BD in the kidney's MRI images that contain measuring of the similarity betwixt the shapes signified by their features. It is computed centred on the segmented blob portion's area as of the image's total area that is articulated as,

$$
S F=\frac{\omega_{b s}^{\prime \prime}}{\omega_{t s}^{\prime \prime}}
$$

Here, $\omega_{b s}^{\prime \prime}$ signifies the segmented blob portions' area; $\omega_{b s}^{\prime \prime}$ implies the image's total area. Lastly, the features extracted are arithmetically formulated as:

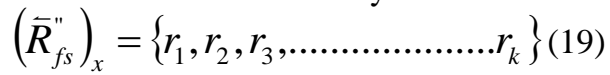

Here, $\left(\bar{R}_{f s}^{\prime \prime}\right)_{x}$ implies the extracted feature set that is fed as input into the classifier and $r_{k}$ signifies the $k$ number of features prevalent in the extracted feature set.

\subsection{Classification}

Past the FE, classification is executed employs the feature extracted as input. The classification's intent is the categorizing of every pixel present on an image to one amidst the numerous classes. The technique pursued in the categorization is centred on the EDCNN algorithm, which is utilized for categorizing '4' classes: normal classes, glomerulonephritis classes, stone classes, and also Pyelonephritis classes. In a normal convolutional neural network (CNN) algorithm, every feature is pondered for examining the classification outcome. Therefore, it consumes added time and even offers low accuracy. Aimed at evading time and increasing the prediction accuracy, the proposed system utilizes CNN with intense learning neural network. Nevertheless, for incrementing the prediction accuracy level, the $\mathrm{L}_{\mathrm{P}}$-pooling is utilized in the pooling layer that efficiently decreases the computational burden. So, the system employs an Exponential Linear Units (ELU) activation function that is utilized aimed at incrementing the prediction accuracy. Figure 2 exhibits the proposed EDCNN algorithm's working diagram, 


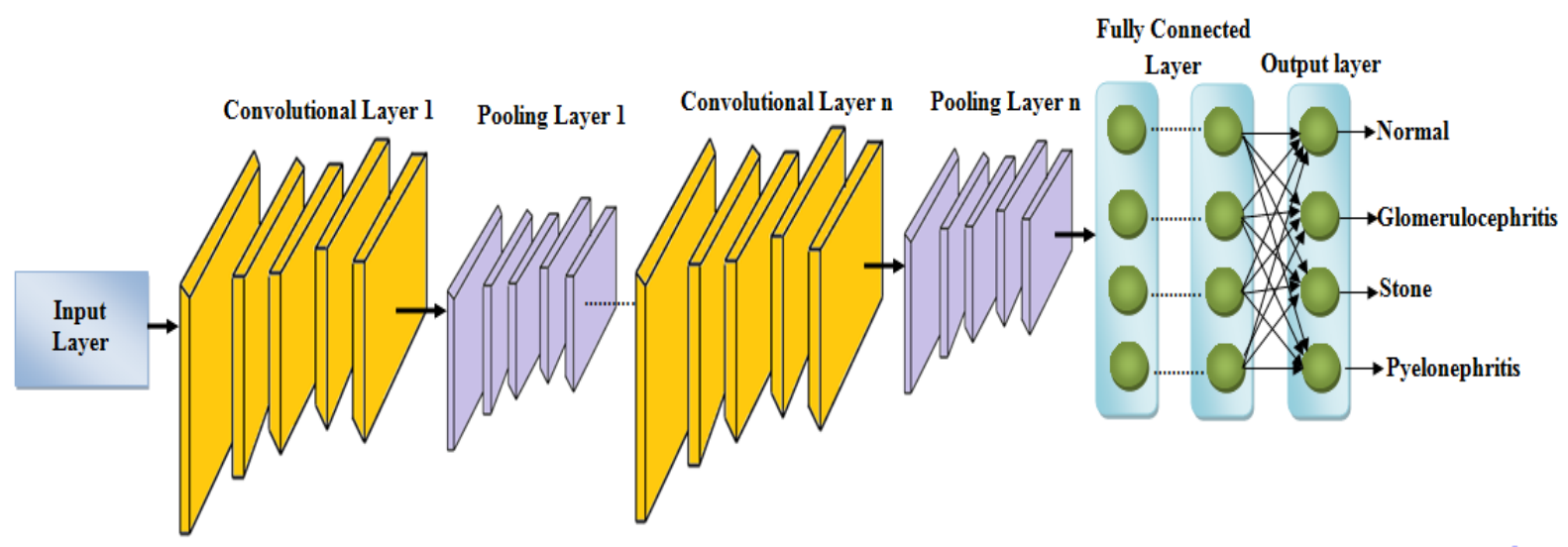

Figure 2: Architecture diagram for the EDCNN algorithm

The EDCNN algorithm's steps are detailed as:

Step 1: Convolution operation

The convolution layer is accountable aimed at calculating the dot layer (comprising nonlinear activation function $\left.\left(\kappa_{a f}\right)\right)$ is enumerated as the product betwixt the weights of the neuron and the region of the inputted image. The convolution's feature map,

$\left(\bar{S}_{c l}^{\prime \prime}\right)_{x}=\kappa_{a f}\left(\left(\bar{R}_{f s}^{\prime \prime}\right)_{x}\left(\bar{T}_{w v}^{\prime \prime}\right)_{x}\right)(20)$

Here, $\left(\bar{S}_{c l}^{\prime \prime}\right)_{x}$ signifies the convolution layer's output; $\left(\bar{R}_{f s}\right)_{x}$ implies the inputted value (i.e. extracted feature set); $\left(\bar{T}_{w v}\right)_{x}$ symbolizes the weight values aimed at their inputs.

Step 2: Pooling operation

This operation is implemented usually subsequent to a convolution process. The usual pooling procedures are: mean pooling, average pooling, and also max pooling. It lessens the computational burden by decrementing the number of connections betwixt the convolutional layers. The $\mathrm{L}_{\mathrm{P}}$ pooling is employed for efficient the pooling operation's execution.

$\left(\bar{U}_{p l}^{\prime \prime}\right)_{x}=\left[\sum\left(\bar{S}_{c l}^{\prime \prime}\right)_{x}\right]^{1 / \alpha}(21)$

Here, $\left(\bar{U}_{p l}^{\prime \prime}\right)_{x}$ signifies the pooling operation's output and especially, whilst $\alpha=1$, LP correlated with average pooling; whilst $\alpha=\infty$, LP decreases to max pooling.

Step 3: Next, the filtered combination features are fed as input into the Deep Neural Network (DNN). At first, the inputted feature's weights as of the CNN are arbitrarily presumed. The hidden node's output is obtained by the summing the product of the inputted value and all the inputted nodes' weight vector associated to it. The hidden layer's output is calculated as:

$\left(\bar{V}_{h l}^{\prime \prime}\right)_{x}=\sum\left(\left(\bar{U}_{p l}^{\prime \prime}\right)_{x}\right) \cdot\left(\bar{T}_{w v}^{\prime \prime}\right)_{x}+B i(22)$

Here, $\left(\bar{V}_{h l}^{\prime \prime}\right)_{x}$ signifies the hidden layer's output and $B i$ implies the bias value.

Step 4: Next, the outputted layer is computed as:

$$
\begin{aligned}
& \left(\bar{W}_{o t}^{\prime \prime}\right)_{x}=\sum_{x=1}^{N}\left(\bar{V}_{h l}^{\prime \prime}\right)_{x}\left[\bar{X}_{E L U}^{\prime \prime}\right] \cdot\left(\bar{T}_{w v}^{\prime \prime}\right)_{x}+B i(23) \\
& \bar{X}_{E L U}^{\prime \prime}=\max \left(\left(\bar{V}_{h l}^{\prime \prime}\right)_{x}, 0\right)+\min \left(\eta\left(e^{\left(\bar{V}_{h l}^{\prime \prime}\right)_{x}}-1\right), 0\right)_{(24)}
\end{aligned}
$$

Here, $\left[\bar{X}_{E L U}^{\prime \prime}\right\rfloor$ implies the activation function, $\eta$ symbolizes the predefined parameter aimed at controlling the value to which an ELU saturate aimed at the negative inputs; $\left(\bar{W}_{o t}^{\prime \prime}\right)_{x}$ implies the outputted value. The figure below exhibits the EDCNN algorithm's pseudo-code, 
Small Blob Detection and Classification in 3D MRI Human Kidney Images Using IMBKM and EDCNN Classifier

Input: Extracted feature set $\left(\bar{R}_{f_{2}}\right)_{\alpha}=\left\{r_{1}, r_{2}, r_{3}, \ldots \ldots \ldots \ldots \ldots . . . . r_{k}\right\}$
Output: Classified Blobs

Begin

Initialize $\left(\bar{S}_{t I}^{*}\right)_{x}$ be the convolution layer's output, $\left(\bar{T}_{w}^{-\prime}\right)_{x}$ be the weight value, $\left(\bar{U}_{x \mid}^{\prime \prime}\right)_{x}$ be the pooling layer output, $\left(\bar{V}_{k=1}^{*}\right)_{x}$ and $B i$

Calculate the number of training samples

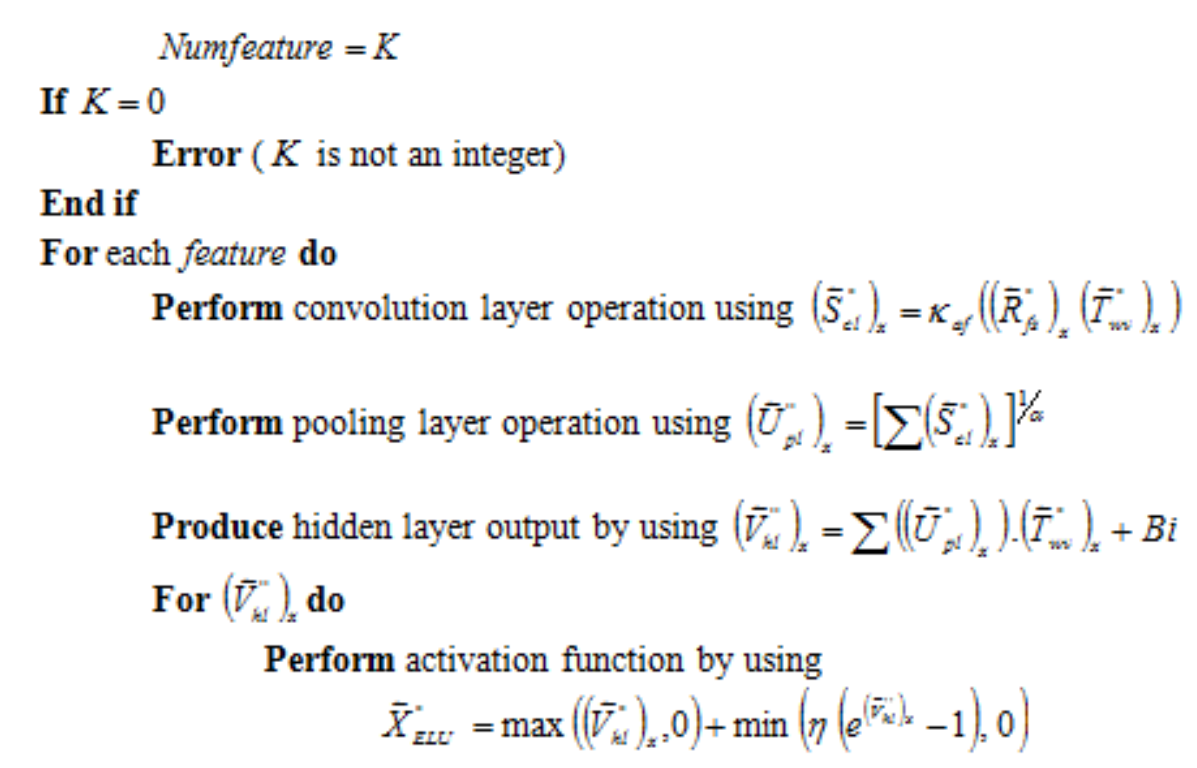

Compute the output layer output

End for

End for

End

Figure 3: Pseudo-code for the EDCNN algorithm

\section{RESULTS AND DISCUSSIONS}

Here, the performance of the proposed small blob detection and classification in 3D-MRI human kidney images using IMBKM and EDCNN classifier is proffered. In Python, the execution of proposed blob detection along with classification is done. The system is applied to real 3D-MRI human kidney images for assessing the proposed method's performance and it gathers images as of the openly assessable datasets. Performance analysis of segmentation as well as classification is the two ways in which the proposed system's performance is analyzed. Below subsection elucidates the performance analogy in details.

\subsection{Performance Analysis of Segmentation}

Here, the proposed IMBKM algorithm's performance is analogized with the various prevailing methods namely MBKM, Fuzzy C-Means (FCM), K-Means, along with Active Contour (AC) algorithms. Centred on various qualitative performance metrics say, Negative Predictive Value (NPV), sensitivity, specificity, accuracy, precision, f-measure, along with Positive Predictive Value (PPV), the performance analogy is done and this performance are analyzed as of the below table. 
Table 1: Comparison of blob detection performance

\begin{tabular}{|l|l|l|l|l|l|}
\hline Metrics & $\begin{array}{l}\text { Proposed } \\
\text { IMBKM }\end{array}$ & MBKM & K-Means & FCM & $\begin{array}{l}\text { Active } \\
\text { Contour }\end{array}$ \\
\hline Accuracy & 99.09 & 84.47 & 55.25 & 59.72 & 51.25 \\
\hline Sensitivity & 99.96 & 84.02 & 60.87 & 58.51 & 57.59 \\
\hline Specificity & 92.17 & 91.32 & 32.72 & 49.72 & 3.17 \\
\hline Precision & 99.66 & 51.37 & 43.45 & 17.28 & 5.6 \\
\hline F-Measure & 95.74 & 58.68 & 32.71 & 24.58 & 1.23 \\
\hline NPV & 0.9893 & 0.9854 & 0.8462 & 0.9455 & 0.8165 \\
\hline PPV & 0.9966 & 0.5139 & 0.0434 & 0.1728 & 0.0052 \\
\hline
\end{tabular}

Discussion: Centred on the accuracy, precision, sensitivity, specificity, NPV, f-measure, along with PPV metrics, the above table exhibits the proposed model's segmentation performance with the prevailing MBKM, K-Means, FCM, along with AC methods. The proposed IMBKM's performance is very high when analogized with the prevailing method is evinced on analyzing the table. For instance, $99.09 \%$ of accuracy is proffered by the proposed IMBKM whereas the prevailing MBKM, K-Means, FCM, along with AC algorithms achieves an accuracy of $84.47 \%, 55.25 \%, 59.72 \%$, along with $51.25 \%$, which is very lesser when analogized with the proposed IMBKM. Similarly, $57.59 \%$ of sensitivity, $3.17 \%$ of specificity, $5.60 \%$ of precision, $1.23 \%$ of $\mathrm{f}-$ measure, $0.8165 \%$ of NPV, along with of PPV $0.0052 \%$ value is attained by the prevailing AC algorithm. But the proposed IMBKM offers $99.96 \%$ of sensitivity, $92.17 \%$ of specificity, $99.66 \%$ of precision, $95.74 \% \mathrm{f}-$ measure, $0.9893 \%$ of NPV, along with PPV $0.9966 \%$ which are high when analogized with the prevailing method. The proposed IMBKM is more effectual for detecting the blobs in kidney images and is evinced as of the tabular analysis. Below figure exhibits the graphical illustration of this analysis.

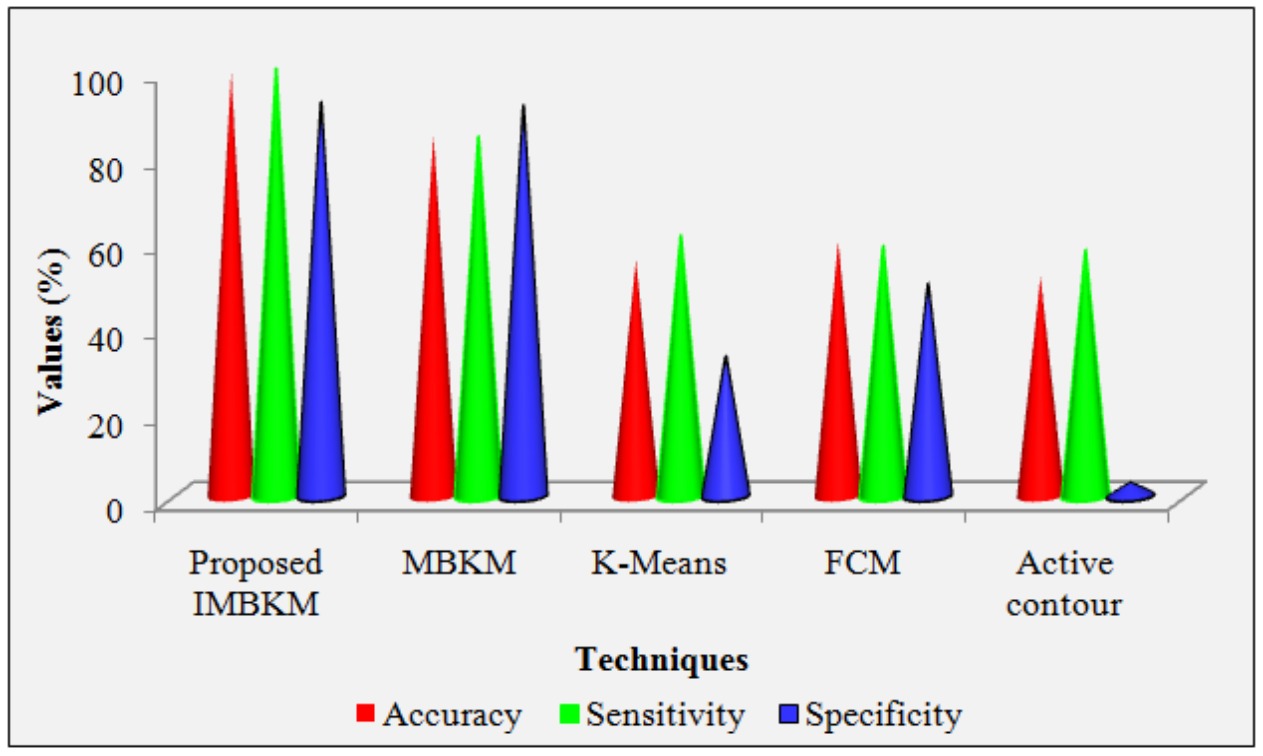

(a) 


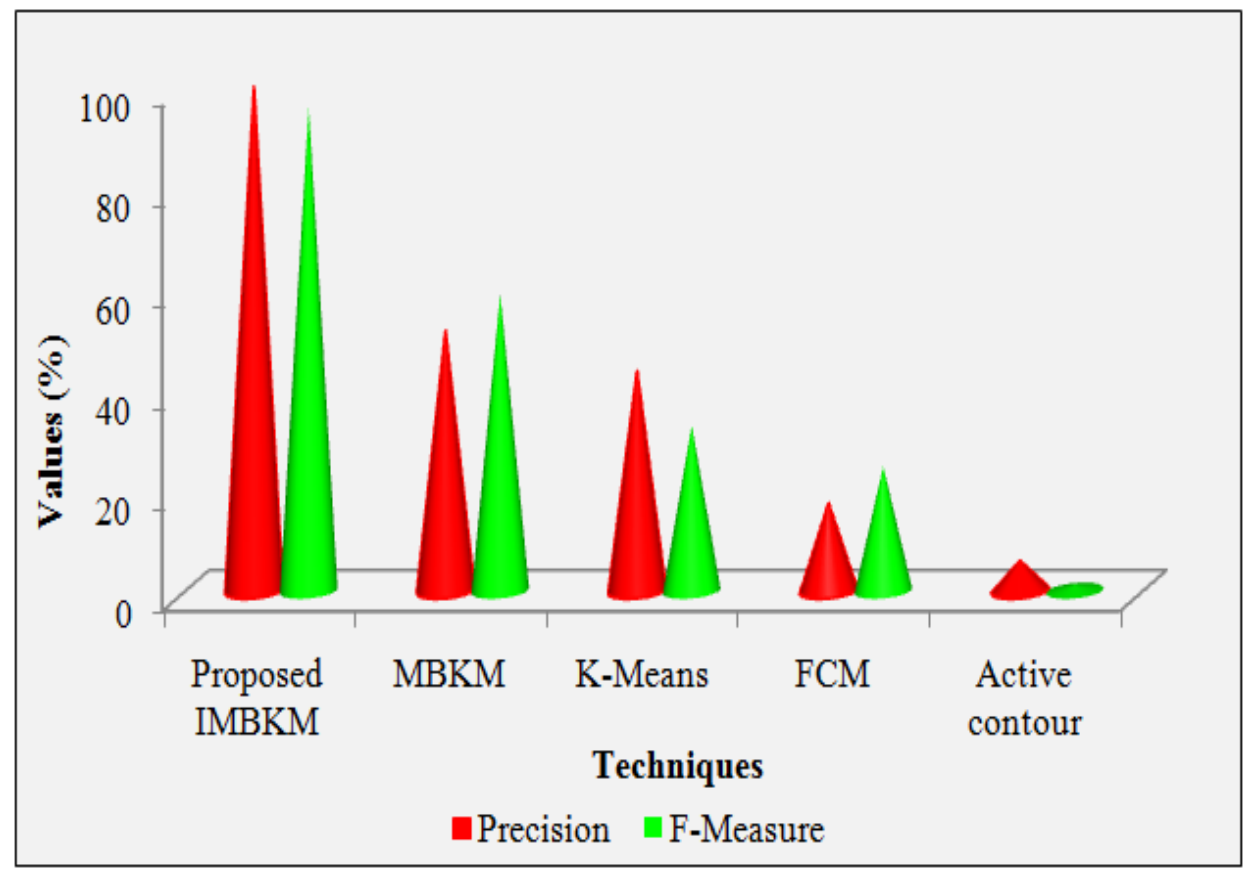

(b)

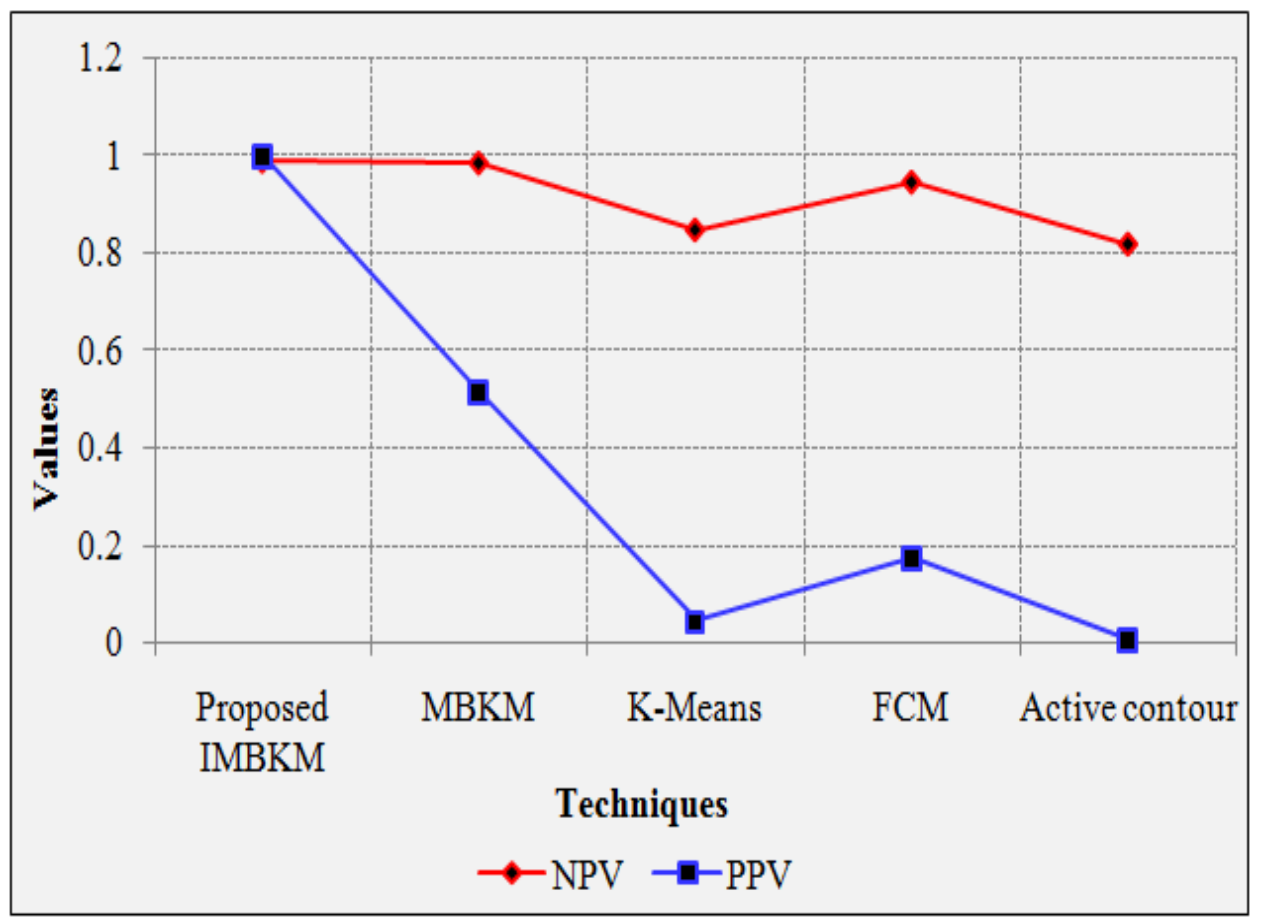

(c)

Figure 4: (a) Accuracy, sensitivity, and specificity, (b) Precision and f-measure, and (c) NPV and PPV based

\subsection{Performance Analysis of Classification}

comparison

Here, the proposed blob classification's performance is analyzed. Centred on the accuracy, specificity, sensitivity, f-measure, False Predictive Rate (FPR), False Negative Rate (FNR), precision, NPV, along with Mathew Correlation Coefficient (MCC) metrics, proposed EDCNN classifier's performance is analogized with the prevailing DCNN, CNN, K-Nearest Neighbour (KNN), along with Naive Bayes (NB) classifiers. Below figure exhibits the proposed EDCNN classifier's Comparative analysis with the prevailing methods. 


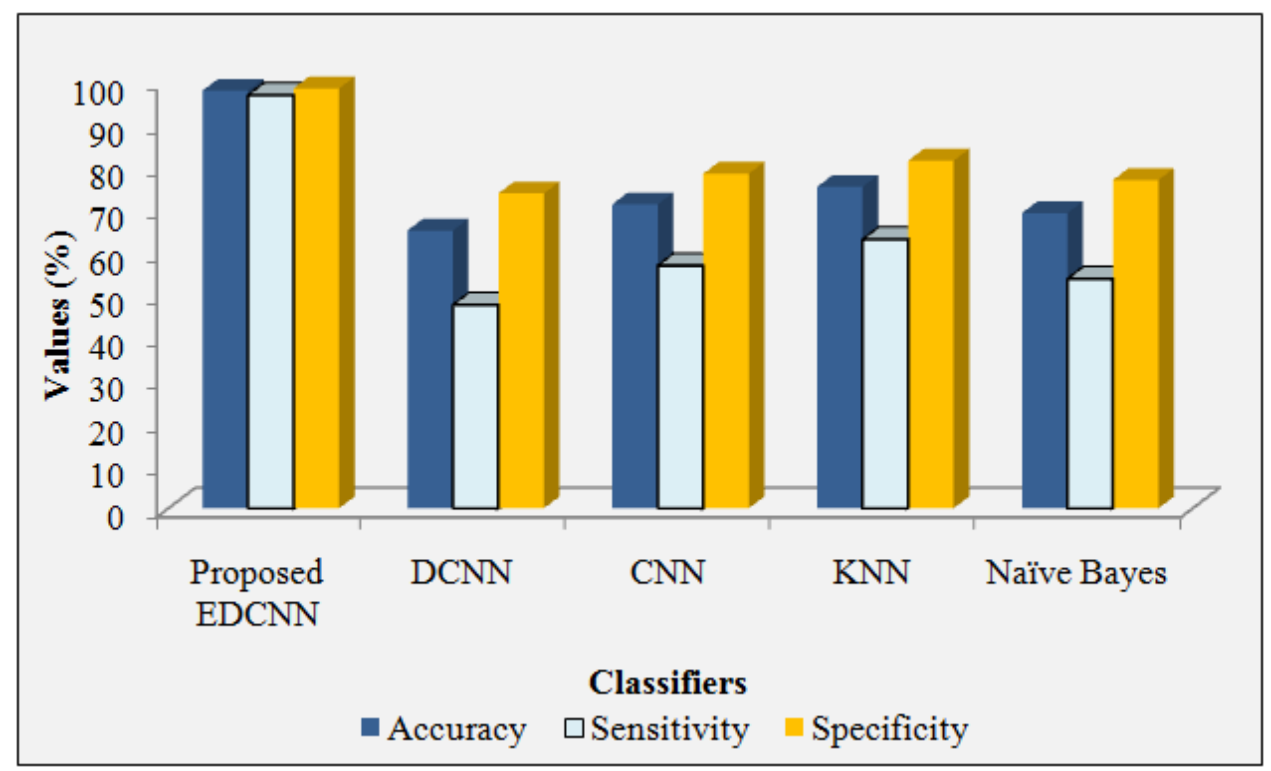

Figure 5: Accuracy, sensitivity, and specificity graph for the proposed EDCNN classifier

Discussion: Centred on the accuracy, sensitivity, together with the specificity metrics, above figure analogizes the proposed EDCNN's performance with the prevailing DCNN, NB, CNN, along with KNN algorithms. The proposed EDCNN detects the blobs most effectually since it achieves accuracy value nearer to $100 \%$ for every blob whereas low detection accuracy outcomes are proffered by the prevailing DCNN, KNN, NB, along with CNN algorithms. For instance, $97.94 \%$ of accuracy, $96.92 \%$ of sensitivity, along with $98.46 \%$ of specificity is proffered by the proposed EDCNN classifier whereas the prevailing method achieves low outcomes concerning accuracy sensitivity, along with specificity. Thus, the proposed EDCNN classifier effectively detects the blobs in kidney images are evinced since it achieves better performance than the various prevailing methods.

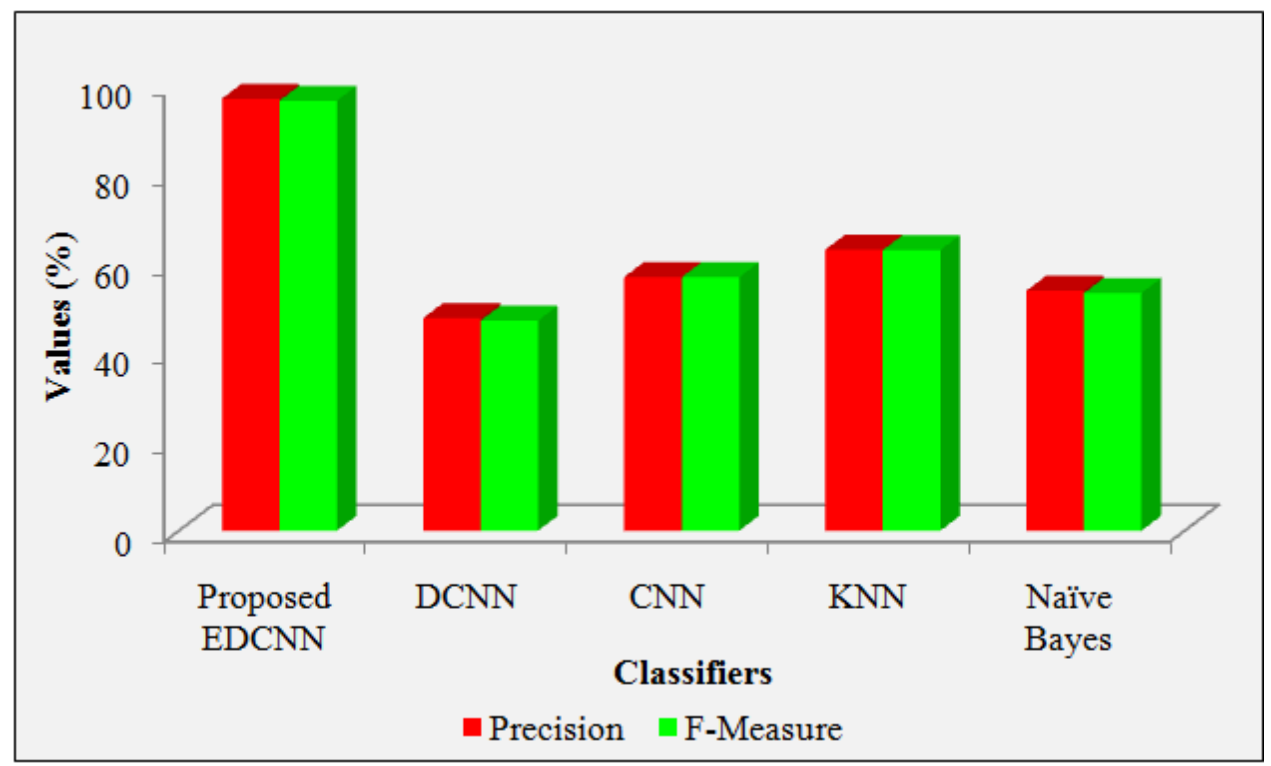

Figure 6: Precision and F-measure based comparison

Discussion:The proposed EDCNN's performance is analogized with the various prevailing techniques say, DCNN, CNN, NB, along with KNN algorithms. Centred on metrics say precision together with f-measure, the performance analogy is executed. The BD system's vital performance metrics are f-measure together with the Precision. This system offers high precision together with f-measure value and it is evinced as of the analysis. The proposed EDCNN attains $96.93 \%$ of precision along with $96.46 \%$ of f-measure, whereas $53.84 \%$ of precision and $53.31 \%$ of f-measure is achieved by the prevailing NB, which is very less when analogized with the proposed EDCNN. For detecting the blobs in kidney images, the EDCNN classifier is superior to the prevailing techniques concerning precision along with f-measure metrics is evinced by the outcomes. 


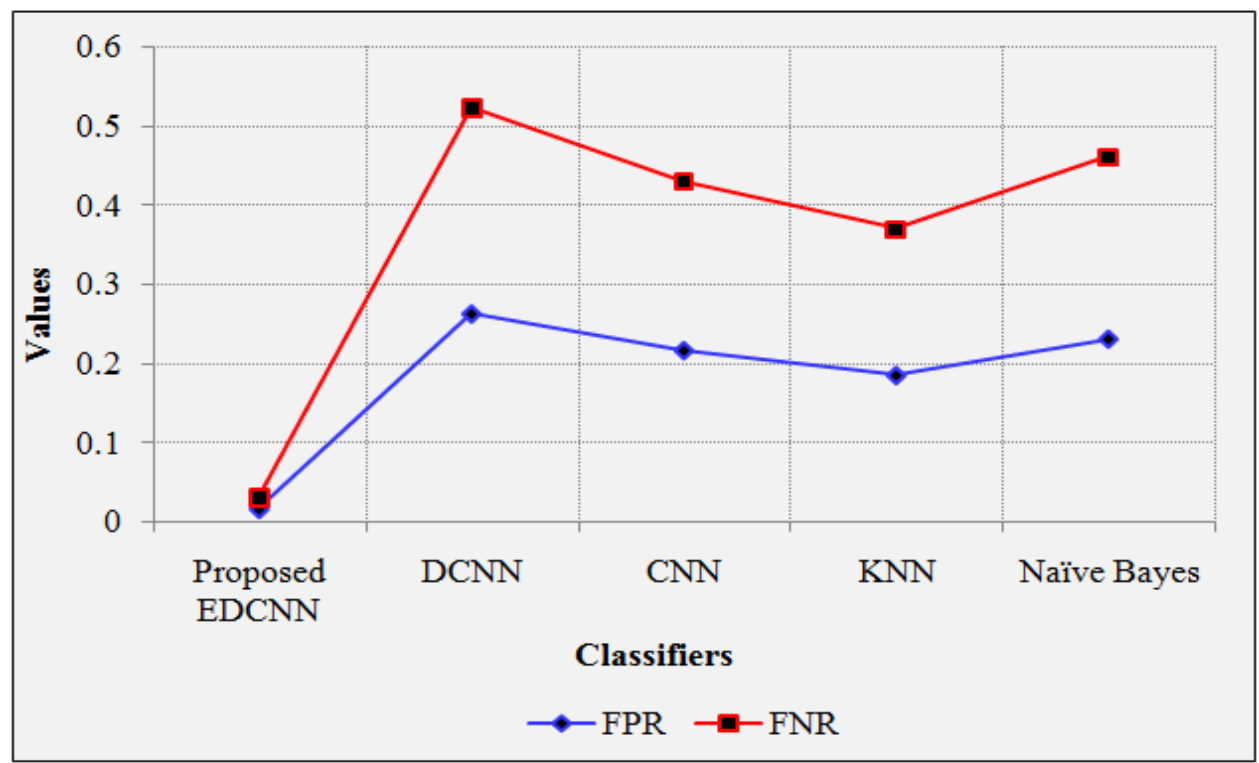

Figure 7: Illustrates the performance of the proposed model with the existent models

Discussion: Regarding the FPR along with FNR metrics, higher performance is attained by the proposed EDCNN when analogized with the prevailing classifiers is evinced in analyzing the above figure. The system could be stated as an enhanced system if it gives better FPR together with FNR values. In this, lower performance is offered by the prevailing NB algorithm when analogized with the proposed EDCNN. Additionally, lower performance is proffered by the prevailing DCNN, CNN, and KNN algorithm. For instance, $0.2302 \%$ FPR and $0.461 \%$ FNR is attained by the prevailing NB classifier whereas the proposed EDCNN classifier attains $0.0158 \%$ of FPR along with $0.0301 \%$ of FNR, which is lesser when analogized with the prevailing classifiers. Thus an encouraging efficacy is offered by the proposed EDCNN when analogized with the prevailing classifiers.

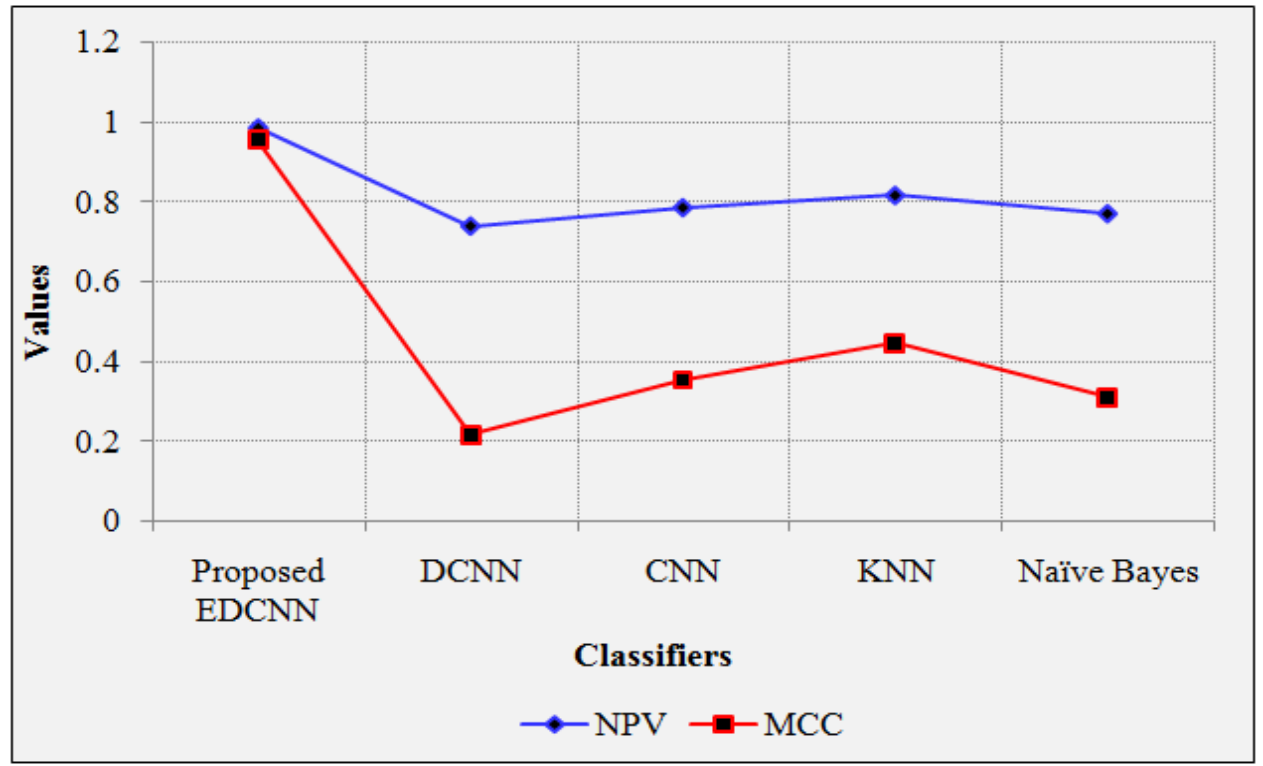

Figure 8: Comparative analysis of the proposed EDCNN classifier with conventional techniques.

Discussion: Centred on NPV along with MCC metrics, the above figure analogizes the proposed EDCNN's performance with the prevailing DCNN, NB, CNN, along with KNN, techniques. Here, the MCC gives a better evaluation than the accuracy when the numbers of negative and positive samples are unbalanced and it takes the value of $(-1,+1)$. Here, NPV of $0.7384 \%$ and $0.2153 \%$ of MCC is achieved by the prevailing DCNN which is less when analogizes with the proposed EDCNN. Likewise, NB of $0.7846 \%, 0.8153 \%$, and $0.7692 \%$ and MCC of $0.3538 \%, 0.4461 \%$, and $0.3076 \%$ is offered by the prevailing CNN, KNN, together with NB respectively. But the proposed EDCNN gives NPV of $0.9846 \%$ and $0.9538 \%$ of MCC, which is superior when analogized with the prevailing techniques. Thus, the proposed EDCNN gives top performance when analogized with the prevailing systems and is evinced as of this analysis. 
Some techniques are proffered for blob detection. An efficient segmentation technique is required aimed at separating the blobs as of the 3D MRI human kidney images for finding the blobs. This work presents proficient algorithms, like IMBKM and EDCNN, aimed at finding the blobs and it categories the diseases as of the blobs utilizing the 3D MRI human kidney images. Initially, for verifying the proposed system's effectiveness, the proposed IMBKM algorithm's performance is examined and also analogized with the traditional MBKM, KMeans, FCM, and also Active contour algorithm centred on a few metrics. Here, the proposed system attains $99.09 \%$ accuracy, $99.96 \%$ sensitivity, $92.17 \%$ specificity, $99.66 \%$ precision, $95.47 \%$ f-measure, $0.9893 \%$ NPV, and also $0.9966 \%$ PPV that is greater than the traditional techniques. After that, the proposed EDCNN Classifier's performance is analogized with the traditional DCNN, CNN, KNN, and Naive Bayes classifiers regarding a few metrics. Here, the proposed system obtains $97.94 \%$ accuracy, $96.92 \%$ sensitivity, $98.46 \%$ specificity, $96.93 \%$ precision, $96.46 \%$ f-measure, $0.0158 \%$ FPR, $0.0301 \%$ FNR, $0.9846 \%$ NPV, and also $0.9538 \% \mathrm{MCC}$ that is also superior to the existent techniques. In general, the outcomes exhibits that the proposed system attains good performance while analogized with the existent methodologies. In the upcoming future, the proposed system's performance can be incremented via the incorporation of added features; feature selection can be executed employing the effectual optimization technique.

\section{REFERENCES}

1. Fei Gao, Teresa Wu, Xianghua Chu, Hyunsoo Yoon, Yanzhe Xu, and Bhavika Patel, "Deep Residual Inception Encoder-Decoder Network for Medical Imaging Synthesis", IEEE journal of biomedical and health informatics, vol. 24, no. 1, pp.39-49, 2019.

2. Angela Giardino, Supriya Gupta, Emmi Olson, Karla Sepulveda, Leon Lenchik, Jana Ivanidze, Rebecca Rakow-Penner, Midhir J. Patel, Rathan M. Subramaniam, and DhakshinamoorthyGaneshan, "Role of imaging in the era of precision medicine", Academic radiology, vol. 24, no. 5, pp. 639-649, 2017.

3. Luke Xie, Kevin M. Bennett, Chunlei Liu, G. Allan Johnson, Jeff Lei Zhang, and Vivian S. Lee, "MRI tools for assessment of microstructure and nephron function of the kidney", American Journal of Physiology-Renal Physiology, vol. 311, no. 6, pp.F1109-F1124, 2016.

4. Hai Ying Zhou, Tian Wu Chen, and Xiao Ming Zhang, "Functional magnetic resonance imaging in acute kidney injury: present status", BioMed Research International, vol. 2016, 2016, 10.1155/2016/2027370.

5. Lulu Xu, Huaxiang Lu, and Min Zhang, "Automatic segmentation of clustered quantum dots based on improved watershed transformation”, Digital Signal Processing, vol. 34, pp.108-115, 2014.

6. AsthaJaiswal, William J. Godinez, Roland Eils, MaikJörg Lehmann, and Karl Rohr, "Tracking virus particles in fluorescence microscopy images using multi-scale detection and multi-frame association", IEEE Transactions on Image Processing, vol. 24, no. 11, pp. 4122-4136, 2015.

7. Xiongwei $\mathrm{Wu}$, Doyen Sahoo, and Steven $\mathrm{CH}$ Hoi, "Recent advances in deep learning for object detection", Neurocomputing, 2020, 10.1016/j.neucom.2020.01.085.

8. Ala'A. R Al-Shamasneh, Hamid A. Jalab, ShivakumaraPalaiahnakote, UnaizahHanumObaidellah, Rabha W. Ibrahim, and Moumen T. El-Melegy, "A new local fractional entropy-based model for kidney MRI image enhancement”, Entropy, vol. 20, no. 5, pp. 344, 2018.

9. Vidyasaraswathi H. N., and M. C. Hanumantharaju, "Review of various histogram based medical image enhancement techniques", In Proceedings of the International Conference on Advanced Research in Computer Science Engineering \& Technology (ICARCSET 2015), pp. 1-6, 2015, 10.1145/2743065.2743113.

10. Raghunandan, K. S., PalaiahnakoteShivakumara, Hamid A. Jalab, Rabha W. Ibrahim, G. Hemantha Kumar, Umapada Pal, and Tong Lu, "Riesz fractional based model for enhancing license plate detection and recognition", IEEE Transactions on Circuits and Systems for Video Technology, vol. 28, no. 9, pp.2276-2288, 2017.

11. Hamid A.Jalab, Rabha W. Ibrahim, and Amr Ahmed, "Image denoising algorithm based on the convolution of fractional Tsallis entropy with the Riesz fractional derivative", Neural Computing and Applications, vol. 28, no. 1, pp.217-223, 2017.

12. Kay Thwe Min Han, and BunyaritUyyanonvara, "A survey of blob detection algorithms for biomedical images", In IEEE 7th International Conference of Information and Communication Technology for Embedded Systems (IC-ICTES), pp. 57-60, 2016, 10.1109/ICTEmSys.2016.7467122.

13. Yanzhe Xu, Fei Gao, Teresa Wu, Kevin M. Bennett, Jennifer R. Charlton, and Suryadipto Sarkar, "U-net with optimal thresholding for small blob detection in medical images", In IEEE 15th International Conference on Automation Science and Engineering (CASE), pp. 1761-1767, 2019, 10.1109/COASE.2019.8843234. 
14. Gang, Carlos Lopez-Molina Wang and Bernard De Baets, "Automated blob detection using iterative Laplacian of Gaussian filtering and unilateral second-order Gaussian kernels", Digital Signal Processing, vol. 96, pp. 102592, 2020.

15. Xiaopeng Xu, "Blob detection with the determinant of the Hessian", In Chinese Conference on Pattern Recognition, Springer, Berlin, Heidelberg, pp. 72-80, 2014, 10.1007/978-3-662-45646-0_8.

16. Hongming $\mathrm{Xu}$, Cheng Lu, Richard Berendt, NareshJha, and Mrinal Mandal, "Automatic nuclei detection based on generalized laplacian of gaussian filters", IEEE Journal of Biomedical and Health Informatics, vol. 21, no. 3, pp.826-837, 2016.

17. Gang Wang, Carlos Lopez-Molina, and Bernard De Baets, "Blob reconstruction using unilateral second order Gaussian kernels with application to high-ISO long-exposure image denoising", In Proceedings of the IEEE International Conference on Computer Vision, pp. 4817-4825, 2017, 10.1109/ICCV.2017.516.

18. Min Zhang, Teresa Wu, Scott C. Beeman, Luise Cullen-McEwen, John F. Bertram, Jennifer R. Charlton, Edwin Baldelomar, and Kevin M. Bennett, "Efficient small blob detection based on local convexity, intensity and shape information", IEEE transactions on medical imaging, vol. 35, no. 4, pp.1127-1137, 2015.

19. Yanzhe Xu, Teresa Wu, Fei Gao, Jennifer R. Charlton, and Kevin M. Bennett, "Improved small blob detection in 3D images using jointly constrained deep learning and Hessian analysis", Scientific reports, vol. 10, no. 1, pp. 1-12, 2020.

20. Min Zhang, Teresa Wu, and Kevin M. Bennett, "Small blob identification in medical images using regional features from optimum scale", IEEE transactions on biomedical engineering, vol. 62, no. 4, pp. 1051-1062, 2014.

21. Min Zhang, Teresa Wu, and Kevin M. Bennett, "A novel Hessian based algorithm for rat kidney glomerulus detection in 3D MRI", In Medical Imaging 2015: Image Processing, International Society for Optics and Photonics, vol. 9413, p. 94132N, 2015,10.1117/12.2081484.

22. Fei Gao, Min Zhang, Teresa Wu, and Kevin M. Bennett, "3D small structure detection in medical image using texture analysis", In IEEE 38th annual international conference of the Engineering in Medicine and Biology Society (EMBC), pp. 6433-6436, 2016, 10.1109/EMBC.2016.7592201.

23. Hui Kong, HaticeCinarAkakin, and Sanjay E. Sarma, "A generalized Laplacian of Gaussian filter for blob detection and its applications", IEEE transactions on cybernetics, vol. 43, no. 6, 1719-1733, 2013. 